\title{
Pearson Correlation Coefficient
}

National Cancer Institute

\section{Source}

National Cancer Institute. Pearson Correlation Coefficient. NCI Thesaurus. Code C65172.

A measure of the correlation of two variables $X$ and $Y$ measured on the same object or organism, that is, a measure of the tendency of the variables to increase or decrease together. It is defined as the sum of the products of the standard scores of the two measures divided by the degrees of freedom. 